\title{
tic\&société
}

Vol. 13, No 1-2 | 1er semestre 2019 - 2ème semestre 2019

Les industries culturelles à la conquête des

plateformes?

\section{Le spot TV à l'heure des plateformes. Une analyse de la stratégie publicitaire du Groupe TF1}

Alexandre JOUX

\section{(2) OpenEdition}

Journals

Édition électronique

URL : http://journals.openedition.org/ticetsociete/3406

DOI : 10.4000/ticetsociete.3406

Éditeur

Association ARTIC

Édition imprimée

Pagination : 295-322

Référence électronique

Alexandre JOUX, «Le spot TV à l'heure des plateformes. Une analyse de la stratégie publicitaire du

Groupe TF1 », tic\&société [En ligne], Vol. 13, № 1-2 | 1er semestre 2019 - 2ème semestre 2019, mis en ligne le 20 avril 2019, consulté le 15 mai 2019. URL : http://journals.openedition.org/ticetsociete/3406 ; DOI : 10.4000/ticetsociete.3406 
tic\&société - 13(1-2), 2019

\title{
Le spot TV à l'heure des plateformes. Une analyse de la stratégie publicitaire du Groupe TF1
}

\begin{abstract}
Alexandre JOUX
Alexandre Joux, Aix Marseille Univ, Université de Toulon, IMSIC, Marseille, France, est maître de conférences en sciences de l'information et de la communication, chercheur à I'IMSIC et directeur de l'École de journalisme et de communication d'Aix-Marseille (EJCAM). Ses recherches portent sur l'économie de l'information et des industries culturelles, en particulier dans les contextes de convergence et d'internationalisation, et sur les évolutions du journalisme dans son rapport aux environnements médiatiques et numériques. alexandre.joux@univ-amu.fr
\end{abstract}


Le spot TV à l'heure des plateformes. Une analyse de la stratégie publicitaire du Groupe TF1

\section{Le spot TV à l'heure des plateformes. Une analyse de la stratégie publicitaire du Groupe TF1}

Résumé : Cet article met en lumière la plateformisation du marché publicitaire sous l'influence des moteurs de recherche et des réseaux sociaux numériques. II analyse la manière dont les médias tentent de s'y adapter en mixant data management et brand safety, ce qui nous permet de mettre en évidence des logiques de coopétition entre plateformes et médias. L'article analyse ensuite la stratégie de la régie du Groupe TF1, première régie "média » de France. Cette stratégie mobilise les atouts de la publicité sur Internet pour mieux valoriser l'offre publicitaire télévisée. Elle fait du spot TV sur les chaînes à forte audience un élément clé de toute campagne de communication, ce que ne peuvent pas proposer les régies des acteurs de l'Internet. Mais cette course à la taille, sur Internet comme à la télévision, pose la question de l'avenir des médias à l'audience plus confidentielle quand ils se financent avec la publicité.

Mots-clés : publicité sur Internet, spot TV, plateformes, Gross Rating Point (GRP), brand safety.

Abstract: This article highlights the platformization of the advertising market as influenced by search engines and social networks. It analyzes how the media try to adjust by mixing data management and brand safety, highlighting coopetition strategies between platforms and media industries. The article examines the strategy of the TF1 group's advertising department, the first "media" advertising department in France, which is based upon using the advantages of Internet advertising to better promote the television advertising offer. It makes TV spot advertising on large audience channels a key element of any communication campaign, something that the advertising departments of Internet companies cannot offer. But this race to critical size, on both the Internet and television, raises questions concerning the future of small audience media who depend on advertising revenues.

Keywords: Online advertising, TV spot, platforms, Gross Rating Point (GRP), brand safety. 
Resumen: Este artículo estudia el movimiento de plataformización del mercado publicitario bajo influencia de los motores de búsqueda y de las redes sociales. Analiza el proceso de adaptación de los medios como consecuencia de la utilización del data management y de la brand safety, lo que permite mostrar lógicas de coo-petición entre las plataformas y los medios de comunicación. En primer lugar, se analiza la estrategia de la división publicitaria del Grupo televisivo TF1, la empresa publicitaria más importante de Francia. Dicha estrategia moviliza las ventajas de la publicidad en Internet para poner en valor a oferta publicitaria televisiva. Consigue que el spot televisivo en los canales de grandes audiencias sea un elemento clave de cualquier campaña de comunicación, lo cual no pueden proponer las empresas publicitarias en Internet. Pero esta carrera al tamaño, tanto en Internet como en la televisión plantea la cuestión del futuro de los medios de comunicación en relación con la privacidad.

Palabras clave: publicidad en Internet, anuncio televisivo, plataformas, Gross Rating Point (GRP), brand safety. 
Le spot TV à l'heure des plateformes. Une analyse de la stratégie publicitaire du Groupe TF1

Depuis 2017, chaque présentation des résultats du marché publicitaire français se traduit par une salve de commentaires sur la domination de Google et de Facebook, qu'il s'agisse des présentations du marché média dans son ensemble ou du seul marché publicitaire en ligne. Pour celui-ci, la source de référence est le Syndicat des régies Internet (SRI) qui publie un observatoire en association avec I'Udecam (Union des entreprises de conseil et achat média). Fédérant d'abord des régies en ligne, on pourrait s'attendre à une certaine indulgence à l'égard des deux géants de l'Internet de la part du SRI. Or, c'est tout le contraire: le SRI rappelle inlassablement que le marché publicitaire en ligne, devenu en 2016 le premier poste de dépenses des annonceurs dans les médias, voit sa croissance essentiellement captée par Google et Facebook, notamment parce que cette croissance dépend de la publicité programmatique et de la publicité sur mobile (SRI, 2018, "Tendances clés »).

Ces deux «plateformes » seraient donc en train d'asseoir leur domination sur la publicité en ligne ; une révolution serait en cours, qui verrait la publicité abandonner les médias au profit de ces nouveaux intermédiaires, puissants parce qu'ils maîtrisent des écosystèmes en ligne et récoltent grâce à eux des masses considérables de données sur leurs utilisateurs (Benzoni et Clignet, 2016). Les " plateformes " seraient ainsi en train d'étouffer l'une des sources historiques de financement des médias, la publicité, menaçant de ce fait la possibilité d'un accès libre aux contenus.

Ces inquiétudes, légitimes, méritent toutefois d'être nuancées. Les médias sont certes en concurrence avec les plateformes quand ils cherchent à se financer grâce à la publicité en ligne, mais ils développent aussi des relations plus complexes avec ces mêmes plateformes. C'est ce que nous nous proposons d'analyser ici, en nous concentrant sur le média qui attirait jusqu'en 2015 les plus importantes dépenses des annonceurs, à savoir la télévision. 
Depuis la crise économique de 2008, le marché publicitaire de la télévision parvient à résister tout à la fois à la concurrence d'Internet et à la tendance baissière des dépenses des annonceurs (BUMP, 2018). Cette résistance du marché publicitaire de la télévision s'explique par les atouts propres au média audiovisuel, mais également par sa capacité à s'approprier les logiques des plateformes.

Afin d'explorer ces enjeux, nous analyserons dans un premier temps le rôle des plateformes en ligne sur le marché publicitaire pour montrer que leur domination va bien au-delà de leurs parts de marché. Cette domination se manifeste d'abord dans l'imposition à l'ensemble du marché de critères d'évaluation de la performance publicitaire. À cet égard, nous mobiliserons la notion de plateformisation pour décrire la manière dont cette mesure de la performance publicitaire essaime bien au-delà des seules régies des plateformes en ligne, au point de devenir la référence partagée par l'ensemble du marché.

Dans un deuxième temps, nous nous questionnerons sur cette plateformisation du marché publicitaire, notamment pour les médias. En effet, grâce à leurs contenus, ceux-ci sont essentiels pour les plateformes en ligne, d'où la notion de coopétition pour décrire leurs relations. Cette coopétition rappelle l'intérêt des contenus des médias pour les annonceurs, qui se lit dans les chiffres du marché publicitaire en ligne sur des segments spécifiques, ainsi de l'IPTV.

À cet égard, il s'agira de s'interroger sur la nature de la relation entre médias et plateformes en ligne. Les médias sontils dans un rapport de subordination à l'égard des plateformes en ligne ? Ou cette coopétition signale-t-elle la possibilité d'une alternative? Sur l'autre versant du marché, les annonceurs voient en effet dans les contenus des médias la garantie de la brand safety ("sécurité de marque ", le terme français étant très peu utilisé). Or celle-ci devient essentielle à mesure que la publicité programmatique se développe et optimise l'affichage publicitaire indépendamment du contexte éditorial environnant.

Pour tenter d'apporter une première réponse à cette interrogation, nous nous pencherons dans un dernier temps sur les interrelations complexes entre plateformes et média télévisé à partir d'une étude monographique. En effet, toute tentative de 
Le spot TV à l'heure des plateformes. Une analyse de la stratégie publicitaire du Groupe TF1

généralisation semble ici risquée tant diffèrent les stratégies des chaînes et les moyens qu'elles sont susceptibles de déployer. C'est pourquoi nous nous concentrerons sur la seule stratégie du Groupe TF1, et ce, pour plusieurs raisons. D'une part, il abrite encore la plus importante régie plurimédias de France, qui fut longtemps leader avant que les annonceurs ne donnent l'avantage à Google. Les activités de régie du Groupe TF1 sont donc les premières menacées par la concurrence nouvelle des formats publicitaires en ligne. D'autre part, le Groupe TF1 est très peu développé dans le secteur de la télévision payante. II doit donc impérativement préserver ses recettes publicitaires et défendre, auprès des annonceurs, la valeur de la communication publicitaire à la télévision. Or, à l'inverse de la publicité personnalisée sur Internet, la communication publicitaire sur un média généraliste et linéaire dispose d'avantages qui semblent à l'opposé de ceux mis en avant par les plateformes lorsqu'elles prônent de nouvelles manières de mesurer l'efficacité publicitaire. Le Groupe TF1, en tant que leader de la publicité à la télévision, est donc en première ligne face aux logiques des plateformes en ligne, avec une stratégie dont on verra qu'elle repose sur une adaptation de son offre au marché publicitaire en ligne pour mieux valoriser, en retour, son offre de spots à la télévision.

En ce qui concerne la méthodologie, nous procéderons par une étude documentaire, dans la mesure où le terrain considéré fait l'objet de publications professionnelles de référence. Pour l'analyse du marché publicitaire, les publications du SRI seront principalement mobilisées ${ }^{1}$. Pour l'analyse de l'offre publicitaire du Groupe TF1, nous nous appuierons sur les "conditions commerciales » de la régie TF1 Publicité ${ }^{2}$ et sur la revue Références du Groupe TF1,

\footnotetext{
${ }^{1}$ Le SRI, Syndicat des régies Internet, publie de manière récurrente un Observatoire de l'e-pub, réalisé par le cabinet pwc qui enquête auprès des principales régies en ligne en France. Les chiffres de l'Observatoire de l'e-pub sont la référence du marché, l'Observatoire de l'e-pub étant d'ailleurs publié en partenariat avec l'Udecam. Nous mobiliserons principalement le bilan 2017 de l'Observatoire de l'epub, publié en janvier 2018 et dressant la synthèse des derniers chiffres disponibles lors de la rédaction de cet article (SRI, 2018).

${ }^{2}$ Les médias qui ne recourent pas à des enchères en ligne pour la commercialisation de leurs espaces mettent à disposition du marché les tarifs de leurs espaces avant négociation. C'est le cas de la régie TF1 Publicité, qui publie pour chacun de ses supports (chaîne TF1, TNT/Thématiques, Digital, etc.) à la fois des "conditions commerciales ", à savoir un document présentant la nature de l'offre publicitaire, ainsi que des "conditions générales de vente »(CGV), lesquelles précisent aux
} 
éditée à destination des " professionnels du marketing et de la communication » (Références, 2017, p. 34), notamment son numéro "Spécial data » de janvier 2017. S'ajoute à cela une importante revue de presse permettant de mobiliser les déclarations des représentants du Groupe TF1, sachant que ces déclarations doivent toujours être considérées comme relevant à la fois de l'information et de la communication. Les références scientifiques sur lesquelles repose cette étude relèvent d'une part des sciences économiques et de gestion, d'autre part des sciences de l'information et de la communication, avec un thème fédérateur, la stratégie des plateformes, un champ d'études ouvert en 2002 dans l'ouvrage fondateur d'Annabelle Gawer et Michael Cusumano (2002).

\section{La plateformisation du marché publicitaire}

Nous avons jusqu'ici employé le terme plateforme sans recourir à une définition scientifique précise. C'est qu'il n'en existe pas de stabilisée. Ainsi, Gawer et Cusumano (2014) notent l'« ubiquité » du terme (p.418) mobilisé tant dans le champ du management, de la technologie que de l'économie industrielle. Ils inscrivent toutefois l'étude des plateformes dans les sciences de gestion et les sciences économiques, lesquelles caractérisent les plateformes "par la complémentarité d'un noyau de composants "essentiels" et stables auquel s'adjoint une multitude de composants périphériques et variables " (Bullich, 2018, p. 4).

Cette approche des plateformes, élaborée dès la fin des années 1980, s'éloigne de l'acception dominante du terme depuis que celui-ci a été associé au développement de l'intermédiation sur Internet. En effet, au-delà du champ de l'économie industrielle et de l'innovation, le terme plateforme sert aussi à désigner les grands acteurs de l'économie numérique qui s'imposent comme des intermédiaires entre une offre et une demande. II désigne également le dispositif

annonceurs les conditions de la contractualisation avec la régie ainsi que les indices tarifaires pratiqués. Dans cet article, nous mobiliserons d'abord les conditions commerciales de TF1 Publicité parce que leur promesse permet de décrypter la stratégie de la régie. Publiées en octobre 2017, les Conditions commerciales 2018 ont servi de référence, notamment celles de la chaîne TF1 (TF1, 2017a), ainsi que les Conditions commerciales Digital 2018 (TF1, 2017b) et les Conditions commerciales 2018 TNT/Thématiques (TF1, 2017c). 
Le spot TV à l'heure des plateformes. Une analyse de la stratégie publicitaire du Groupe TF1

d'intermédiation en tant que tel. D'ailleurs, cette dénomination est revendiquée par les plateformes en ligne (Gillespie, 2010), qui mobilisent le terme plateforme pour développer une relation positive avec leurs utilisateurs, les annonceurs, leurs clients, mais également à destination des pouvoirs publics afin de bénéficier d'une régulation spécifique favorable.

Reste que les deux acceptions du terme plateforme ne sont pas antinomiques. Les plateformes en ligne incarnent aujourd'hui des stratégies qui ont été explorées par d'autres firmes auparavant, Internet ne faisant que favoriser le déploiement accéléré des logiques d'intermédiation sur de nouveaux marchés. À cet égard, si l'on peut aisément distinguer les plateformes en ligne des industries culturelles parce que les premières obligeraient les secondes à dépendre de leurs services pour toucher les internautes (voir, pour le cas de la presse, Bell et Owen, 2017), il n'en reste pas moins que les industries culturelles peuvent également déployer des stratégies de type " plateforme », jouant autrement la carte de l'intermédiation entre une offre et une demande.

Ces stratégies, qui relèvent des logiques de plateforme, mais sont déployées par des acteurs historiques, non issus d'Internet, sont relativement peu étudiées. À l'évidence, leurs conséquences sur les marchés sont bien moindres que celles des plateformes en ligne. Pourtant, mobiliser les logiques de plateforme permet de construire une grille d'analyse pertinente pour aborder les stratégies d'entreprise qui visent à faire émerger un écosystème contrôlé susceptible de générer des formes nouvelles d'intermédiation. C'est ce que nous nous proposons ici d'appliquer à l'offre publicitaire du Groupe TF1, en mobilisant d'abord non pas le terme de plateforme, mais celui de plateformisation pour désigner le processus par lequel une entreprise va chercher à construire un écosystème au sein duquel elle contrôlera le composant "essentiel ». En la matière, la plateformisation du marché publicitaire par la régie TF1 se traduit par l'émergence de modalités nouvelles de valorisation des audiences afin de proposer une alternative à l'offre des plateformes en ligne, Google et Facebook en premier lieu.

Le choix du terme plateformisation indique qu'il s'agit bien de stratégies d'entreprise, donc d'un processus évolutif qui n'implique pas l'identification hic et nunc d'une nouvelle 
plateforme. Nous le mobilisons ici pour sa valeur heuristique, en ce qu'il permet d'identifier des comportements d'entreprise. Nous ne nous limitons donc pas à son acception dominante, qui relève d'abord du discours d'accompagnement de la numérisation de l'économie. La plateformisation est, en effet, souvent entendue comme la capacité d'un acteur numérique à investir un marché contrôlé par des acteurs historiques pour le désintermédier, ainsi que le fait Uber sur le marché des taxis, Airbnb sur le marché de l'hébergement touristique, ou encore Blablacar sur les transports longue distance.

Ce processus de plateformisation est au cœur des évolutions du marché publicitaire. Traditionnellement séparé en marché média et hors média, le marché publicitaire a connu en vingt ans une mutation sans précédent, notamment le marché média. Celui-ci repose sur l'appariement d'une offre d'espaces dans les médias (presse, radio, télévision, cinéma, affichage) à une demande de la part des annonceurs (Gabszewicz et Sonnac, 2006). Les médias financés par la publicité relèvent ainsi d'un marché à deux versants, où le "médium » et son audience sont vendus aux annonceurs, quand les contenus du " médium » sont adressés aux publics (Anderson et Gabszewicz, 2005). En conséquence, les régies des médias commercialisent d'abord une audience constituée en fonction du contexte éditorial, ce qui leur permet de vendre des espaces premium de communication. Elles s'engagent en outre à garantir un nombre d'expositions au message publicitaire. À la télévision, ce critère quantitatif de mesure se traduit dans le GRP (Gross Rating Point, ou point de couverture brut), qui mesure le nombre moyen de contacts publicitaires pour cent individus de la cible visée.

Transposé sur Internet, ce modèle commercial a donné naissance aux premières offres de display (les bannières graphiques, fixes puis vidéo) qui sont des encarts au sein de pages web proposant un contenu éditorial. Parce qu'il s'agit d'un décalque en ligne de la publicité média, les bannières ont été historiquement vendues au coût pour mille (CPM). Le site web garantit une audience, mesurée en nombre de visiteurs uniques ou de pages vues, donc un score de type quantitatif, l'annonceur étant responsable de la performance de sa communication. Dès lors, l'annonceur va procéder classiquement. Il élabore un plan média qui le conduira à 
Le spot TV à l'heure des plateformes. Une analyse de la stratégie publicitaire du Groupe TF1

privilégier certains sites web à d'autres, parce que l'audience qu'ils fédèrent, en rapport avec leur offre éditoriale, se rapproche le plus possible des cibles visées par la campagne de communication.

Ce modèle de valorisation volera en éclat avec l'émergence de mesures nouvelles de la performance publicitaire en ligne, lesquelles vont même s'imposer comme l'élément clé de l'écosystème publicitaire en ligne et rendre possible sa plateformisation. Plusieurs facteurs ont contribué à leur émergence.

Le premier est incontestablement le passage d'un univers de rareté dans les médias à un univers d'abondance dans l'offre de contenus en ligne. À partir du moment où l'offre d'espaces publicitaires déborde largement la demande des annonceurs sur Internet, le prix des encarts baisse et incite les régies à imaginer des formes nouvelles de valorisation des bannières. $\mathrm{Ce}$ phénomène favorisera l'émergence du ciblage comportemental au début des années 2000 aux États-Unis grâce à la collecte massive de données personnelles (Ouakrat, 2012). Les bannières sont encore vendues au coût pour mille, mais les régies vont s'engager autrement auprès des annonceurs. Au lieu de commercialiser un contexte éditorial et les audiences associées, elles vont commercialiser des profils d'utilisateurs et la connaissance de leurs attentes sur le moment. Le message publicitaire est désormais décorrélé du contenu éditorial auquel il était historiquement associé pour être affiché cette fois-ci en fonction des seuls individus cibles, quelle que soit la nature des contenus qu'ils consultent. Dès lors, le contexte éditorial est neutralisé et toute page web devient un support pertinent de communication personnalisée. Les gigantesques inventaires des sites (l'ensemble des espaces publicitaires d'un éditeur, y compris les pages web à très faible audience) deviennent exploitables à la condition d'automatiser les procédés d'affichage des publicités. La valorisation des audiences auprès des annonceurs va donc dépendre de la capacité des acteurs à récolter et à exploiter les données des utilisateurs et à maîtriser l'affichage en temps réel des bannières en fonction de leurs profils (Evans, 2009). II s'agit de la publicité dite "programmatique », parce que des programmes (des algorithmes) automatisent la totalité du processus de ciblage et d'affichage. Concrètement, peu de 
régies média disposent de telles compétences et elles doivent déléguer à des intermédiaires spécialisés la valorisation de leurs encarts publicitaires par des données d'utilisateurs, ces intermédiaires captant l'essentiel de la valeur sur le marché publicitaire display (Lallement, Serris et Varenne, 2014).

Le second phénomène est à l'origine exogène au marché publicitaire des bannières, puisqu'il s'agit de la rupture imposée par Google avec les liens sponsorisés. Parce que les pages de résultats du moteur de recherche n'accueillaient à l'origine aucun contenu éditorial (ce n'est plus le cas aujourd'hui), mais y renvoyaient grâce à des liens hypertextes, Google a opté pour un format publicitaire adapté à l'interface de son service. Plutôt que de vendre des encarts graphiques, il a vendu l'affichage de liens hypertextes en haut des résultats de recherche afin que les annonceurs puissent bénéficier d'une visibilité que le référencement naturel ne garantit pas nécessairement. Pour l'internaute, le message publicitaire et l'information recherchée sur Google Search ont la même apparence, un lien hypertexte, ce qui a l'avantage d'éviter le phénomène de pollution visuelle par la publicité. La publicité devient un service rendu à l'internaute, les liens sponsorisés étant d'ailleurs affichés en fonction de leur score de qualité. Si l'annonceur est responsable de son score de qualité parce que ce dernier croise taux de clic et pertinence des mots-clés achetés, il n'est plus responsable stricto sensu de la performance de sa campagne de communication: son message éditorial n'est plus affiché, comme ce serait le cas avec une bannière. C'est donc au moteur de garantir l'affichage du bon lien hypertexte face à la bonne personne (Evans, 2008). Pour y parvenir, Google dispose d'un avantage incommensurable: ses utilisateurs renseignent chacune de leurs demandes en tapant leur requête. La dimension participative du moteur de recherche permet ainsi à Google Search de garantir une publicité dite "contextuelle ", à savoir garantir que l'internaute cherche bien ce type de lien hypertexte sur le moment.

Cette forme de ciblage comportemental permettra à Google de proposer des modalités nouvelles de commercialisation des espaces publicitaires. Parce que Google est garant de l'efficacité de la communication publicitaire, il ne peut pas s'engager uniquement sur un taux minimum d'affichage des 
Le spot TV à l'heure des plateformes. Une analyse de la stratégie publicitaire du Groupe TF1

annonces, dans une logique d'audience associée au coût pour mille, mais il doit s'engager à ne facturer que les liens sponsorisés effectivement cliqués, ce qui correspond à une facturation au coût par clic (CPC). Cette publicité facturée à la performance va ainsi permettre aux annonceurs de mesurer précisément leur retour sur investissement (ROI), puisqu'ils peuvent suivre sur leur site web le comportement des utilisateurs apportés par leur campagne de liens sponsorisés ${ }^{3}$. Dès lors, les modalités de commercialisation de la publicité peuvent être transformées en profondeur. À la place des agences d'achat d'espaces, qui servent d'intermédiaires entre annonceurs et éditeurs sur le modèle des agences de courtage, Google substitue une place de marché où les annonceurs décident du prix qu'ils sont prêts à payer pour chaque clic associé à un mot-clé. Le coût du mot-clé va s'aligner sur le taux de marge moyen des secteurs d'activité des annonceurs parce qu'il est indexé sur la mesure du ROI.

La facturation de la publicité à la performance s'imposera ensuite sur l'ensemble du marché publicitaire en ligne, le ciblage comportemental et la publicité programmatique n'étant que le moyen trouvé par le marché display pour garantir ce que Google Search propose avec la facturation au coût par clic. C'est en ce sens que nous parlons d'une plateformisation du marché publicitaire en ligne : ce sont désormais les régies de Google et Facebook qui donnent le « la » pour l'ensemble du marché publicitaire. Parce qu'elles disposent de masses considérables de données sur leurs utilisateurs, elles garantissent une performance publicitaire qui oblige les éditeurs de contenus en ligne à s'aligner sur leur modèle, soit en passant par leurs régies, soit en cherchant à atteindre une taille critique d'utilisateurs et une expertise forte en data management afin de proposer une offre publicitaire compétitive, une stratégie initiée par exemple par le Groupe Figaro dans le domaine de l'information (Joux, 2017).

\footnotetext{
${ }^{3}$ Voir le centre d'aide de Google Ads pour le fonctionnement des enchères et le calcul du ROI : https://support.google.com/google-ads/answer/2375454?co= ADWORDS.IsAWNCustomer\%3Dfalse\&hl=fr (consulté le 8 janvier 2019).
} 
Alexandre JOUX

\section{Des logiques de coopétition entre plateformes et médias ?}

Vente à la performance et ciblage comportemental renversent le modèle historique de facturation de la communication publicitaire par les régies, qui reposait sur l'association entre contenu des médias et message publicitaire, et sur un engagement en termes d'audience. À cet égard, les médias étaient entre eux complémentaires, cette complémentarité s'illustrant dans la notion de plan média. Avec la profusion des offres en ligne, vente à la performance et ciblage comportemental remplacent les plans média en rendant possible l'exploitation des gigantesques inventaires publicitaires en ligne. Ils favorisent ainsi la plateformisation du marché publicitaire en ligne parce qu'ils donnent un avantage concurrentiel décisif aux acteurs capables de récupérer le plus grand nombre de données sur leurs utilisateurs, et parce qu'ils étendent à l'ensemble des publications en ligne la possibilité d'accueillir une communication publicitaire performante.

Ces acteurs, notamment Google et Facebook, imposent tout à la fois leur modèle de valorisation des espaces à l'ensemble du marché et leurs régies face à celles des médias en ligne. En effet, parce qu'ils réduisent en grande partie les asymétries informationnelles entre annonceurs et éditeurs de contenus grâce à leur maîtrise des données des utilisateurs, ces acteurs s'imposent comme des intermédiaires essentiels sur le marché publicitaire en ligne. Cette intermédiation favorise les situations monopolistiques dans la mesure où la compétition dans la fourniture de services d'intermédiation sur un marché à deux versants dégrade la qualité du service fourni, puisque les firmes concurrentes vont pratiquer la rétention des données. En cas de compétition, "des stratégies de différenciation forte sont pratiquées par les plateformes, ce qui [...] ramène au cas monopolistique $^{4}$ » (Brousseau et Penard, 2007, p. 91). Ainsi Facebook est-il spécialisé dans les bannières et une valorisation des encarts grâce à des données sociales, alors que Google est historiquement positionné sur les liens sponsorisés et une valorisation reposant sur des données contextuelles.

${ }^{4}$ Traduction libre de : «strong differentiation strategies are practiced by platforms, which brings [us] back to the monopolistic case ». 
Le spot TV à l'heure des plateformes. Une analyse de la stratégie publicitaire du Groupe TF1

Cette plateformisation du marché publicitaire en ligne se traduit par l'importance des dépenses des annonceurs sur Internet au détriment des autres médias, ce qui révèle l'efficacité des modèles publicitaires en ligne, compétitifs sur le plan tarifaire et garants de performance. Mais cette plateformisation du marché publicitaire pose problème aux médias. Les situations monopolistiques qu'elle génère conduisent à une captation de la valeur par les plateformes en ligne, avec le risque d'une évolution de type "winner-takesall » et " loser-gets-nothing » (Shapiro et Varian, 1999, p. 188). Or les médias savent que, sur un marché à deux versants, ce sont eux, avec leurs contenus, qui rendent possible l'audience que les intermédiaires en ligne valorisent sur le plan publicitaire en enrichissant chaque contact d'une couche d'informations comportementales. En ce sens, plateformes et médias s'inscrivent dans une logique de coopétition. Les médias utilisent les outils des plateformes pour valoriser leurs espaces publicitaires en ligne, ce qui permet aux plateformes de disposer en contrepartie d'une offre enrichie de contenus, un phénomène constaté notamment entre infomédiaires et éditeurs de presse en ligne (Rebillard et Smyrnaios, 2010). Cette coopétition, en valorisant les contenus des médias, notamment les contenus de qualité (premium), permet également aux médias de mieux résister à la concurrence des autres éditeurs de sites web. Certes, les espaces de ces derniers se retrouvent dans les inventaires des régies au même titre que ceux des médias, mais il n'en demeure pas moins que l'attention des utilisateurs est plus forte pour les contenus éditorialisés et les publicités qui leur sont associées (Cho, 1999).

L'étude des chiffres du marché publicitaire conduit ainsi à une position plus nuancée sur la domination des plateformes qui trahit la place importante occupée encore par les médias classiques disposant de contenus premium. Publié par France Pub, l'IREP (Institut de recherches et d'études publicitaires) et Kantar Media, le baromètre unifié du marché publicitaire 2017 révèle une bonne résistance de la télévision, dont les recettes publicitaires nettes sont en hausse de $1 \%$, à 3,286 milliards d'euros, grâce notamment à la forte progression des recettes issues du parrainage (BUMP, 2018). Les espaces classiques, à savoir les spots, sont en revanche en léger repli, à - 0,3\%, un phénomène finalement positif parce que l'année 2017 n'était 
pas favorable. L'élection présidentielle et la campagne qui la précède favorisent l'attentisme des annonceurs, et l'absence de grands évènements sportifs susceptibles de soutenir la dépense publicitaire rend défavorable la comparaison avec 2016. Autant dire que la télévision s'en sort bien en 2017, ce qui n'est pas le cas de la radio $(-2,6 \%)$ et de la presse $(-7,4 \%)$. Internet, en revanche, s'en sort bien mieux, avec une hausse de ses recettes publicitaires de $12 \%$, à 4,094 milliards d'euros. En synthèse, le marché des médias hors revenus numériques s'affiche en repli de 2,3\% en 2017 (BUMP CP, 2018), ce qui rend encore plus notable la progression de $1 \%$ de la télévision. Celle-ci parvient à profiter de la hausse des dépenses en communication des annonceurs malgré l'arbitrage de ces derniers en faveur d'Internet.

En première lecture, le constat semble donc sans appel: alors que les recettes publicitaires des médias baissent, la télévision semble à peu près résister, quand la part dévolue à Internet augmente au détriment des autres supports. C'est d'ailleurs le premier constat dressé par l'étude du Syndicat des régies Internet sur le marché publicitaire en ligne : « En 2017, le digital accroît son avance sur les autres médias » (SRI, 2018, p. 6). La conclusion de l'étude est, là encore, sans équivoque. Les deux principaux postes de dépense publicitaire en ligne, le search et le display, sont captés principalement par deux acteurs: Google et Facebook. Ensemble, «le search et les réseaux sociaux représentent $78 \%$ du marché display et search réunis »(SRI, 2018, p. 41). Reste donc $22 \%$ pour les autres supports et acteurs, les sites des médias, mais aussi tous les sites web qui se financent grâce à la publicité sans pour autant produire de contenus. Ce déséquilibre entre search et réseaux sociaux numériques, d'une part, et les autres acteurs de l'Internet, d'autre part, tend par ailleurs à s'accentuer puisque $92 \%$ de la croissance du marché du search et du display sont captés par les seuls moteurs de recherche et les réseaux sociaux (SRI, 2018).

D'autres tendances méritent pourtant d'être notées. Entre 2015 et 2017, les recettes de l'IPTV, à savoir les spots vidéo diffusés sur les services de replay des chaînes, ont tout simplement doublé, passant de 30 millions d'euros à 60 millions d'euros (SRI, 2018). Les chiffres sont encore faibles comparés aux recettes de la publicité à la télévision, mais ils témoignent 
Le spot TV à l'heure des plateformes. Une analyse de la stratégie publicitaire du Groupe TF1

de la capacité des chaînes à trouver en ligne les moyens de se développer. Cette note d'optimisme traverse en fait l'étude du SRI, qui amorce en conclusion la possibilité d'une évolution des arbitrages des annonceurs quant à l'allocation de leur dépense publicitaire. Certes, "le critère de prix reste souvent le plus important » (SRI, 2018, p. 38), mais, et c'est la toute dernière phrase de l'étude, " la multiplication des points de contact et la complexification du parcours d'achat questionnent le modèle d'attribution historique au last click. De nouvelles formes de mesures sont à l'étude pour mieux redistribuer la valeur entre les différents acteurs » (p. 43).

La domination des moteurs de recherche et des réseaux sociaux numériques sera donc nuancée si d'aventure les nouvelles formes de mesure donnent l'avantage aux contenus des médias et si les annonceurs changent leurs pratiques. C'est ce qu'il faut lire entre les lignes de l'étude du SRI. L'année 2017 a en effet été marquée par la publicisation des inquiétudes des plus grands annonceurs mondiaux, qui ont dénoncé l'association de leurs messages à des contenus problématiques sur les plateformes, menaçant ainsi leur image de marque (problématique de brand safety). Ce phénomène est lié au succès de la publicité programmatique, qui affiche les publicités en ligne en fonction des profils des internautes et non en fonction du contexte éditorial propre au site web qui accueille l'annonce. Pour répondre aux inquiétudes des annonceurs, les médias ont proposé une alternative aux inventaires publicitaires des plateformes en ligne dont la qualité éditoriale n'est pas garantie. Plusieurs médias français se sont ainsi associés dans des régies communes. Ils ont mutualisé leur offre d'espaces publicitaires en ligne afin de proposer, ensemble, une audience capable de rivaliser sur le plan national avec celles des plus grandes plateformes, tout en garantissant la qualité éditoriale du contexte d'affichage des publicités. En France, deux initiatives ont été annoncées durant l'été 2017: la régie Gravity, qui fédère les espaces et les données associées aux utilisateurs d'un grand nombre de médias et de e-commerçants (Fnac-Darty); et l'alliance des groupes Figaro et Le Monde, dans une offre commune d'espaces, baptisée "Skyline ». Pour celle-ci, les messages des annonceurs sont associés à des contenus éditoriaux dits " premium » et un lectorat relevant majoritairement des classes socioprofessionnelles supérieures $(\mathrm{CSP}+)$ très prisées des 
annonceurs.

Ces initiatives s'inscrivent en réaction à la plateformisation du marché publicitaire en ligne. Parce que les plateformes en ligne disposent d'inventaires gigantesques et de données précises sur leurs utilisateurs, les médias sont contraints de s'associer pour gagner ensemble une taille critique leur permettant de se présenter comme alternative à Google ou Facebook. Ils jouent ensuite de leur avantage concurrentiel, à savoir la qualité de leurs contenus, mais ils le font sur un marché où ce sont les plateformes qui ont imposé leurs règles. D'autres initiatives semblent plus innovantes, quand un groupe intègre la logique des plateformes pour repenser la totalité de son offre, y compris hors ligne, avec l'ambition de définir des critères nouveaux de performance pour le marché publicitaire. C'est le cas du Groupe TF1, qui doit impérativement préserver la pertinence de son offre d'espaces publicitaires à la télévision.

\section{La plateformisation de l'offre publicitaire du Groupe TF1}

Présentée comme une " content marketplace multisupport, multisegment, multiplateforme » (TF1, 2017b, p. 3), la régie du Groupe TF1 insiste d'emblée sur l'environnement éditorialisé des espaces publicitaires qu'elle commercialise. Mais cette régie a ceci de particulier qu'elle donne la priorité aux annonces vidéo afin d'associer systématiquement les chaînes de télévision aux campagnes, y compris quand il s'agit de commercialiser des campagnes en ligne. Ainsi les Conditions commerciales digital 2018 de TF1 Publicité présentent-elles en première page le spectre des audiences du groupe avec 30,5 millions de téléspectateurs par jour sur les différentes chaînes en régie, dont TF1, l'offre TNT du groupe et un univers élargi de chaînes thématiques, 850 millions de vidéos vues par mois et 8,2 millions d'auditeurs radio, la régie de TF1 commercialisant les espaces des Indés Radios et de MFM. La régie touche ainsi deux tiers des Français quotidiennement, ce qui en fait le « $1^{\mathrm{er}}$ média social de France » (TF1, 2017a, p. 6) en couverture jour devant les plateformes en ligne, mais grâce à des leviers très différents, avec la chaîne TF1 en navire amiral et une approche résolument plurimédias.

La télévision reste le média roi : après la présentation de 
Le spot TV à l'heure des plateformes. Une analyse de la stratégie publicitaire du Groupe TF1

l'univers élargi de la régie, les Conditions commerciales digital 2018 passent à la présentation des audiences des cinq chaînes en clair du groupe au premier semestre 2017 (TF1, TMC, TFX, TF1 Séries Films, LCI), montrant que le Groupe TF1 part " à la conquête de nouvelles cibles " (TF1, 2017 b, p. 4), alors que le public de la télévision linéaire est vieillissant. La "ménagère de moins de 50 ans ", désormais dénommée «femme responsable des achats" (FRDA<50), reste le cœur de cible du groupe puisqu'elle représente une part d'audience (PDA) de $32,5 \%$, en hausse de $4 \%$ par rapport au premier semestre 2016 ; les 25-49 ans représentent une PDA de $29,4 \%$, en hausse de $3 \%$. Les plus fortes hausses sont à chercher du côté des cibles prisées des annonceurs, les CSP+ avec une PDA de $27,7 \%$, en hausse de $7 \%$, et les 15-34 ans, autrement dit les millénariaux, avec une PDA de 29,5\%, en hausse de $8 \%$ (TF1, 2017b).

En synthèse, les Conditions commerciales digital 2018 de TF1 Publicité commencent donc par un bilan de la refonte des grilles des chaînes du groupe sous l'impulsion d'Ara Aprikian, directeur général adjoint responsable des contenus à TF1 depuis février 2016, refonte qui a consisté en un « travail de segmentation des marques avec des identités fortes et complémentaires ${ }^{5}$ ». La chaîne généraliste TF1 capte les FRDA, quand TMC et $\mathrm{LCl}$ visent plutôt les CSP+, avec l'émission Quotidien de Yann Barthès, programmée en access prime time sur TMC depuis septembre 2016, laquelle a également contribué au rajeunissement de l'audience de la chaîne. Sur LCl, Yves Calvi a été recruté en 2016 pour officier en access prime time, l'expérience n'ayant duré qu'un an, la tranche horaire étant désormais confiée à David Pujadas. Quant aux millénariaux, TFX (ex-NT1) les a pour cible, ce qui permet de rajeunir l'audience du groupe tout en la diversifiant, et TF1 Séries Films (ex-HD1) joue le même rôle avec une programmation renforcée de séries américaines. Autant dire que l'offre éditoriale des chaînes du Groupe TF1 est pensée comme une plateforme, le socle étant la chaîne TF1, les chaînes satellites faisant partie d'une constellation dont l'identité a été affirmée avec le changement de nom de HD1 et de NT1 en janvier 2018. La chaîne TF1 sert ici de marque-

5 "TF1 renforce l'identité de ses "petites" chaînes », Marina Alcaraz, Les Échos, 10 janvier 2018. 
ombrelle aux autres chaînes parce qu'elle a la « capacité de faire profiter du capital de confiance qu'elle a patiemment constitué à des produits qui essaiment dans son sillage » (Bassoni et Joux, 2014, p. 71).

Si cette stratégie est évidente pour l'offre de chaînes, elle est à nuancer pour l'offre de contenus en ligne, où le Groupe TF1 ne dispose pas des mêmes avantages. II faut ainsi attendre la page 5 des Conditions commerciales digital 2018 de TF1 Publicité pour découvrir l'offre en ligne du groupe, avec là encore la priorité donnée à la vidéo et aux chaînes du groupe. En effet, plutôt que de présenter l'offre display de la régie, une approche classique pour un éditeur de contenus en ligne, les conditions commerciales affichent leur préférence pour « l'offre vidéo de TF1 Publicité ", ses 22 millions de visiteurs uniques et ses 850 millions de vidéos vues (TF1, 2017b, p. 5). Le " digital » vient ainsi compléter l'offre d'écrans à la télévision en s'appuyant sur le replay et le live digital des cinq chaînes en clair du groupe, mais aussi le service dédié à la jeunesse Tfou et l'offre en ligne des Indés Radio. S'ajoute à cela une offre vidéo thématique ciblant les $\mathrm{CSP}+$, avec $\mathrm{LCl}$, Eurosport et Discovery Channel, et surtout une offre à destination des millénariaux. Ici, le phénomène de marque-ombrelle ne joue plus, car la régie de TF1 met en avant des marques différentes : Studio71, XTRA, Twitch. La logique de " portfolio » de marques (Aaker, 2004) semble l'emporter pour tester en ligne de nouveaux modèles éditoriaux sans engager directement la marque TF1.

L'hétérogénéité des marques masque cependant une stratégie intégrée. De même que TFX et TF1 Séries Films visent à rajeunir les audiences du groupe à la télévision, XTRA propose des formats courts audiovisuels, Studio71 est un multichannel network qui fédère des chaînes YouTube de "créateurs " et Twitch est le service vidéo contrôlé par Amazon et spécialisé dans les jeux vidéo. Le public de XTRA, des chaînes du Studio71 comme de Twitch correspond aux millénariaux et reproduit en ligne ce que TFX cherche à développer à la télévision. À l'évidence, le Groupe TF1 compte sur ses audiences en ligne pour offrir aux annonceurs des cibles où il est moins dominant à la télévision, ce qui explique le recours à des marques nouvelles qui ne sont pas associées directement à la marque TF1. De ce point de vue, la 
Le spot TV à l'heure des plateformes. Une analyse de la stratégie publicitaire du Groupe TF1

présentation commerciale de l'offre publicitaire en ligne, avec, dans l'ordre, la présentation de l'audience des chaînes, de l'offre vidéo en ligne et de l'offre display classique, atteste d'une plateformisation originale de l'offre de contenus du Groupe TF1 et de l'offre d'espaces publicitaires associée. Cette offre s'illustre d'ailleurs dans une infographie où les médias gérés par la régie gravitent autour de la chaîne TF1 qui s'impose comme l'actif-cœur de la content marketplace (TF1, 2017b).

Il s'agit d'une véritable rupture stratégique. La chaîne TF1 a dominé le marché publicitaire à la télévision, bénéficiant d'une « prime au leader » (Chantepie et Le Diberder, 2005, p. 61) qui lui a permis de capter jusqu'à la moitié de celui-ci. Son pouvoir de prescription s'est érodé en même temps que l'offre de télévision s'est diversifiée et que la consommation de contenus vidéo en ligne s'est développée. Si le groupe n'a pas cru, dans un premier temps, à la TNT, il s'est depuis adapté à ce nouvel environnement en disposant désormais d'un portfolio cohérent de chaînes. Mais l'âge moyen des téléspectateurs des chaînes du groupe augmente, alors que les jeunes plébiscitent la consommation de contenus vidéo en ligne. Le Groupe TF1 doit donc penser sa chaîne dans ce nouvel écosystème élargi et articuler offre linéaire et services en ligne de médias audiovisuels, publicité à la télévision et publicité sur Internet. Le Groupe TF1 est ainsi contraint de se projeter dans un écosystème qui déborde son marché historique. Le groupe semble confronté au dilemme de l'innovateur (Christensen, 1997) parce qu'il doit développer son offre audiovisuelle en ligne et des formats éloignés des émissions de sa chaîne amirale, au risque de cannibaliser son métier historique. Or il s'agit, pour le Groupe TF1, d'un impératif, à la fois pour toucher de nouveaux publics et pour développer sa capacité à commercialiser de la publicité avec les règles nouvelles qui sont celles des plateformes en ligne. C'est notamment ce que trahit le numéro 106 de Références, le magazine de TF1 Publicité de janvier 2017.

Baptisé "Spécial data », ce numéro annonce d'emblée l'enjeu : " le Groupe TF1 place la data et le digital au cœur de sa stratégie de transformation »(REF, 2017, p. 4). Le lecteur s'attend donc à découvrir une présentation de l'offre en ligne du groupe la plus à même de faciliter la récolte de données sur les utilisateurs. L'éditorial rappelle ainsi que la donnée fait partie de 
l'ADN du groupe puisque, "chaque matin à $9 \mathrm{~h}$, les audiences Médiamétrie sont attendues comme le Saint-Graal » (REF, 2017, p. 5). Sauf qu'il s'agit ici des audiences des chaînes en clair, pas des audiences en ligne. Toutefois, 2016 est présentée comme une année charnière, les données intégrant la stratégie d'ensemble du groupe, et notamment de la régie TF1 Publicité, laquelle est présentée comme en avance sur les autres entités du groupe. Cette régie est donc le foyer de l'innovation, ce qui s'est traduit, dès 2015, par le lancement du programme One Data, à savoir la réunion des différentes activités publicitaires du groupe tirant parti des données pour les intégrer dans une Data Management Platform (DMP) unique. En croisant les informations récupérées d'un utilisateur ou d'un foyer sur les différents services du groupe, la régie va alors mettre en œuvre une nouvelle mesure de la performance publicitaire, le GRP Data.

En recourant au GRP, la régie de TF1 confirme son ancrage dans l'univers de la télévision, en même temps qu'elle s'inscrit dans une logique de ciblage imposée par la plateformisation du marché publicitaire. C'est ce que Sylvia Tassan Toffola, directrice générale déléguée aux opérations commerciales de TF1 Publicité, confirme : "Grâce à la data, notre offre est plus affinée, plus enrichie, plus juste. Elle nous fait passer d'une logique de mediaplanning à audienceplanning" (REF, 2017, p. 7). Autant dire que cette évolution répond à la transformation des formes de valorisation publicitaire que Google a favorisée avec la vente de la publicité à la performance. En effet, "le GRP Data comptabilise non plus des individus, mais des quantités achetées, plus exactement les quantités achetées de marques d'une catégorie générées par les téléspectateurs d'un écran. Pratique, pour prédire quel est l'effet d'une publicité TV sur les ventes " (REF, 2017, p. 13). Après le coût par clic, la publicité TV promet également aux annonceurs de pouvoir mesurer précisément leur retour sur investissement.

Si le GRP Data est une réponse aux offres des régies des plateformes en ligne, il n'en reste pas moins qu'il s'agit d'une mesure de l'efficacité publicitaire à la télévision, et non sur Internet. En revanche, les données exploitées proviennent des sources habituelles de la télévision (les panels consommateurs de Kantar et les audiences de Médiamétrie), mais aussi d'Internet, qui contribue fortement à leur enrichissement par 
Le spot TV à l'heure des plateformes. Une analyse de la stratégie publicitaire du Groupe TF1

l'offre en ligne du Groupe TF1. Ici, Internet renforce l'efficacité publicitaire de la télévision plus qu'il ne la concurrence. II s'agit finalement du cœur de la stratégie de TF1 qui n'innove dans la commercialisation de ses espaces publicitaires que pour mieux défendre le marché historique de la publicité télévisée face aux coups de boutoir de la publicité en ligne.

Cette stratégie fait émerger une espèce nouvelle de plateformisation de l'offre publicitaire, qui relève tout à la fois d'un alignement sur les critères de valorisation des plateformes en ligne et d'une volonté de penser ces critères dans un univers élargi qui intègre les dépenses des annonceurs à la télévision et sur Internet. De ce point de vue, la plateformisation de l'offre publicitaire de TF1 est aussi une forme de résistance aux modèles publicitaires des pure players dominants, cette orientation stratégique se retrouvant chez de nombreux éditeurs de chaînes généralistes (Doyle, 2010). Elle articule délibérément données TV et données en ligne, espaces publicitaires à la télévision et sur Internet pour protéger le cœur de métier du Groupe TF1, lequel fait sa force face à des acteurs comme Google ou Facebook. En effet, une campagne de publicité à la télévision génère des synergies avec l'offre en ligne de l'annonceur grâce à l'effet de notoriété qu'elle produit : " pendant la campagne TV, le search progresse de $25 \%$, les visites directes de $34 \%$. Les applis enregistrement $+80 \%$ de téléchargements et $+33 \%$ d'achats in-app à court terme » (REF, 2017, p. 22), selon les chiffres compilés par TF1. Ces effets sont rendus possibles par la pression publicitaire élevée que seules les grandes chaînes généralistes autorisent, l'addition des audiences plus faibles de la TNT n'étant pas, ici, pertinente " en raison d'une saturation de la couverture » (REF, 2017, p. 23). En définitive, l'offre publicitaire de la régie TF1 est présentée comme plus performante que l'offre des plateformes en ligne, car elle associe télévision et numérique, et elle est plus performante que celle des autres régies des chaînes, car elle s'appuie sur la puissance de la chaîne TF1.

Force est toutefois de constater que le modèle d'affaires retenu reste fragile. En effet, les régies des plateformes en ligne ont cet avantage de proposer des campagnes mondiales, ou au moins à l'échelle d'un continent, ce que la télévision classique, ancrée dans les frontières nationales, est incapable d'offrir. Or les annonceurs apprécient ce type d'offres, 
notamment pour les campagnes des produits standardisés de la grande consommation, lesquelles comptent parmi les plus importants budgets publicitaires au monde. À cet égard, certaines initiatives du Groupe TF1 témoignent de sa volonté de répondre aussi à cet enjeu en décalquant à l'échelle européenne ce qu'il a développé en France. Puissant en France, le groupe ne s'est jamais véritablement internationalisé, sauf avec Eurosport, revendu aujourd'hui. Le Groupe TF1 ne peut donc pas s'appuyer sur un réseau de chaînes en Europe comme peut le faire, par exemple, RTL Group (Bertelsmann). Pour contourner cet obstacle et proposer une offre d'espaces au moins européenne, le Groupe TF1 a opté pour une stratégie d'alliance entre acteurs européens.

Le 12 janvier 2017, TF1 a annoncé s'être accordé avec le groupe allemand ProSiebenSat. 1 et le groupe italien Mediaset. Les trois acteurs mutualisent leurs forces pour développer leur présence numérique européenne sur YouTube, une stratégie qui passe par Studio71, détenu par ProSiebenSat.1 et dans lequel les groupes TF1 et Mediaset ont pris respectivement $6,1 \%$ et $5,5 \%$ du capital. Pour Mediaset, l'objectif de cette alliance est clair : « créer l'opérateur le plus important d'Europe dans le talent digital en étroite synergie avec la télévision généraliste ${ }^{6}$ ». Rien de tel, en effet, que de disposer de chaînes puissantes pour attirer les youtubeurs les plus talentueux, ce qu'une plateforme en ligne ne peut pas nécessairement proposer. Cette alliance permet ainsi au Groupe TF1 de diversifier les formats audiovisuels qu'il exploite et de gagner une présence numérique à l'international.

Six mois plus tard, le 9 juin 2017, les trois groupes s'associaient de nouveau pour lancer une plateforme d'achat d'espaces pour de la vidéo programmatique baptisée European Broadcaster Exchange (EBX). Cette plateforme commercialise les espaces publicitaires vidéo sur les sites de replay des chaînes des partenaires, I'IPTV étant l'un des secteurs les plus dynamiques du marché publicitaire display. La promesse faite aux annonceurs repose sur les contenus de qualité des chaînes, donc la brand safety, sur la présence européenne et sur l'exploitation des données des utilisateurs. Depuis novembre 2017 , la chaîne privée britannique Channel4 a rejoint

${ }^{6}$ AFP. (2017, 12 janvier). « Vidéo en ligne : alliance entre TF1, ProSiebenSat.1 et Mediaset $»$. 
Le spot TV à l'heure des plateformes. Une analyse de la stratégie publicitaire du Groupe TF1

l'alliance des chaînes européennes, complétant ainsi la couverture de la régie paneuropéenne. Chris le May, nommé à la tête d'EBX, ne laisse planer aucun doute sur son objectif :

Aujourd'hui, les grands annonceurs mondiaux regardent les médias à l'échelle européenne, alors que chacune des chaînes de télévision opère sur un marché local. Une régie commune va nous permettre d'avoir accès à ces budgets, qui sont aujourd'hui principalement captés par les GAFA dont surtout Google et Facebook.

La plateformisation de l'offre publicitaire des acteurs de la télévision est donc là aussi soumise aux diktats des plateformes en ligne qui, par leur présence mondiale, ont imposé des modalités transnationales de gestion des contrats de communication. C'est en s'adaptant à ces conditions nouvelles, tout en cherchant à valoriser la puissance de leurs contenus, que les grandes chaînes européennes tentent de préserver leur avantage concurrentiel sur le marché publicitaire.

\section{Conclusion : des géants et des perdants ?}

Captation de la croissance du marché publicitaire en ligne, imposition de modalités nouvelles de valorisation des espaces publicitaires, continentalisation des marchés publicitaires, transfert des investissements des annonceurs du hors-ligne vers Internet: autant de facteurs qui attestent de la plateformisation du marché publicitaire sous l'influence des régies en ligne les plus puissantes. Confrontées à ces évolutions, de surcroît très rapides puisqu'elles se sont produites en une quinzaine d'années, les chaînes de télévision ont dû réagir pour défendre le marché publicitaire télévisé, le plus important avant l'apparition d'Internet. Elles ont développé leur offre de contenus en ligne afin de proposer aussi aux annonceurs des campagnes en format vidéo sur Internet. Cette offre en ligne a surtout été utilisée pour toucher des publics nouveaux. Ainsi, le développement des activités de TF1 Publicité sur Internet doit se lire non pas comme une diversification, mais bien comme un moyen de protéger sa part de marché publicitaire à la télévision : les formats publicitaires

7 «Channel 4 s'allie à TF1 et Mediaset face aux Gafa », Marina Alcaraz, Les Echos, 15 novembre 2017. 
en ligne apportent aux annonceurs les cibles que les spots à la télévision touchent moins, tout en permettant à la régie de mieux connaître ses publics grâce à une collecte facilitée de données comportementales. Mais ce phénomène de complémentarité entre publicité TV et en ligne, où Internet renforce la puissance du média télévisé, ne vaut que pour les chaînes les plus puissantes. La régie TF1 rappelle qu'une campagne sur les seules chaînes de la TNT sature très vite la cible visée. Les chaînes de la TNT comme les contenus en ligne trouvent leur plus grande efficacité publicitaire uniquement dans l'association avec des écrans à très forte audience, ce qui donne aux chaînes historiques un avantage compétitif face aux nouvelles concurrences à la télévision, mais aussi face aux régies en ligne des pure players. Associées à l'échelle européenne, ces chaînes sont même en mesure de s'imposer comme une alternative aux régies de Google ou Facebook.

Cette course à la taille soulève toutefois des questions. Le marché publicitaire est-il en train de se reconfigurer autour de quelques grands médias généralistes, notamment à la télévision, qui resterait ainsi le média roi pour la communication de notoriété ? Les médias à plus faible audience, parce qu'ils sont positionnés sur des niches ou des thématiques, sont-ils voués à perdre l'essentiel de leurs recettes publicitaires si d'aventure ils ne parviennent pas à associer leurs écrans à une offre globale portée par un grand média ? Ils semblent en effet plus menacés que les autres parce que la publicité programmatique rend inutile le ciblage des audiences par les plans média qui, autrefois, leur conféraient un véritable intérêt stratégique. Ou alors la télévision, en basculant progressivement sa distribution sur Internet, finira-t-elle par proposer aussi de la publicité personnalisée et individualisée, prenant acte des nouvelles modalités de valorisation des espaces publicitaires imposées par les grandes régies en ligne ? Mais n'est-ce pas là définitivement conférer le contrôle des marchés publicitaires aux seuls acteurs capables de capter l'essentiel de nos données de navigation, qu'il s'agisse des plateformes en ligne ou des opérateurs de télécommunications qui, en France, prennent en charge la distribution des chaînes ? 
Le spot TV à l'heure des plateformes. Une analyse de la stratégie publicitaire du Groupe TF1

\section{Références}

Aaker, D. A. (2004). Leveraging the Corporate Brand. California Management Review, 46(3), 6-18.

Anderson, S. P. et Gabszewicz, J. J. (2005). The media and advertising: A tale of two-side markets, discussion paper 2005. Louvain-la-Neuve, Belgique : Université catholique de Louvain.

Bassoni, M. et Joux, A. (2014). Introduction à l'économie des médias. Paris, France : Armand Colin.

Bell, E. et Owen, T. (2017, 29 mars). The Platform Press. How Silicon Valley reengineered Journalism. Repéré à https://www.cjr.org/tow_center_reports/platform-press-howsilicon-valley-reengineered-journalism.php

Benzoni, L. et Clignet, S. (2016). The Internet: A New Economic Paradigm for the Advertising Market. Digiwolrd Economic Journal, (104), 33-53.

Brousseau, E. et Penard, T. (2007). The Economics of Digital Business Models: A Framework for Analyzing the Economics of Platforms. Review of Network Economics, 6(2), 81-114.

Bullich V. (2018). La "plateformisation » de la formation. Distances et médiations des savoirs, (21). doi: 10.4000/dms.2096

BUMP (2018). Baromètre unifié du marché publicitaire. Paris, France : France Pub, Irep, Kantar Media.

BUMP CP (2018). Le marché publicitaire 2017. L'activité reprend doucement. Paris, France : France Pub, Irep, Kantar Media.

Chantepie, P. et Le Diberder, A. (2005). Révolution numérique et industries culturelles. Paris, France : La Découverte.

Cho, C. H. (1999). How advertising works on the WWW: Modified Elaboration Likelihood Model. Journal of Current Issues and Research in Advertising, 21(1), 34-50. doi: https://doi.org/10.1080/10641734.1999.10505087

Christensen, C. M. (1997). The innovator's Dilemma. When New Technologies Cause Great Firms to Fail. Boston, MA: Harvard Business School Press. 


\section{Alexandre JOUX}

Doyle, G. (2010). From Television to Multi-plaform: Less from more or more for less?. Convergence: The International Journal of Research into New Media Technologies, 16(4), 431-449.

Evans, D. (2008). The Economics of the Online Advertising Industry. Review of Networks Economics, 7(3), 359-391.

Evans, D. (2009). The Online Advertising Industry: Economics, Evolution, and Privacy. Journal of Economic Perspectives, 23(3), 37-60.

France Pub, Irep, Kantar Media (2018). Le marché publicitaire 2017. Paris, France.

Gabszewicz, J. et Sonnac, N. (2010). L'industrie des médias à l'ère numérique. Paris, France : La Découverte.

Gawer, A. et Cusumano, M. A. (2002) Platform Leadership: How Intel, Microsoft, and Cisco Drive Industry Innovation. Boston, MA : Harvard Business Scholl Press.

Gawer, A. et Cusumano, M. A. (2014). Industry Platforms and Ecosystem Innovation. Journal of Product Innovation Management, (31), 417-433.

Gillespie, T. (2010). The Politics of "Platforms". New Media \& Society, 12(3), 347-364.

Joux, A. (2017). Stratégies de marques et stratégies éditoriales du Groupe Figaro. Du portail à l'écosystème intégré par le data management. Réseaux, (205), 117-143.

Lallement, G., Serris, J. et Varenne, D. (2014). L'émergence des places de marché publicitaires, l'opportunité d'une nouvelle industrie. Annales des Mines - Réalités industrielles, (3), 65-69.

Ouakrat, A. (2012). Le ciblage comportemental, une perte de contrôle des éditeurs sur les données de l'audience. tic\&société, 6(1), 33-55. doi: 10.4000/ticetsociete.1251

Rebillard, F. et Smyrnaios, N. (2010). Les infomédiaires, au cœur de la filière de l'information en ligne. Les cas de google, wikio et paperblog. Réseaux, (160-161), 163-194.

Références (REF) (2017). REF Spécial data, (106). 
Le spot TV à l'heure des plateformes. Une analyse de la stratégie publicitaire du Groupe TF1

Shapiro, C. et Varian, H. R. (1999). Information Rules. A Strategic Guide to the Network Economy. Boston, MA: Harvard Business School Press.

SRI (Syndicat des régies Internet) (2018). Observatoire de l'epub, Bilan 2017, $19^{\text {ème }}$ édition - janvier 2018. Paris, France.

TF1 (2017a). Conditions commerciales 2018 TF1. Paris, France : TF1 Publicité.

TF1 (2017b). Conditions commerciales Digital 2018. Paris, France : TF1 Publicité.

TF1 (2017c). Conditions commerciales 2018 TNT/Thématiques.

Paris, France : TF1 Publicité. 